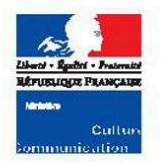

Secrétariat général

Service de la

politiques culturelles

et de l'innovation

Département

des études,

de la prospective
et des statistiques

182, rue Saint-Honoré, 75033 Paris cedex 0

중 0140157917 - 国 0140157999

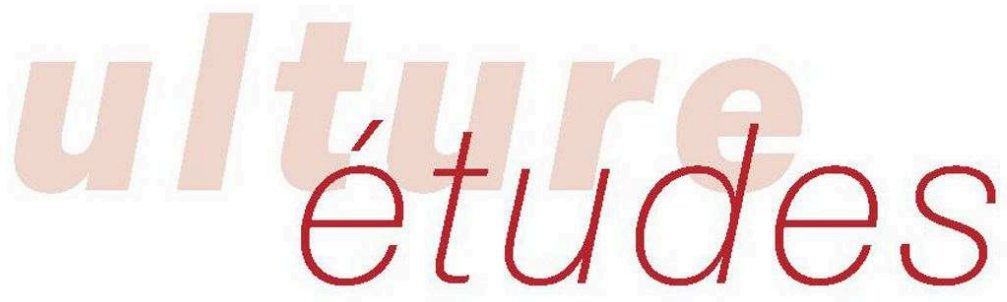

\title{
Médiation culturelle: l'enjeu de la gestion des ressources humaines
}

\author{
Nicolas Aubouin, Frédéric Kletz, Olivier Lenay*

\section{Cultural Promotion: \\ The Human Resource Management Issue}

\begin{abstract}
Avant-propos
Depuis plus de trente ans, les activités de médiation culturelle, qui visent à favoriser l'appropriation collective des différentes formes d'art et de patrimoine, ont connu un essor continu dans l'ensemble des domaines artistiques et culturels. Pour autant, ni ces activités, ni les emplois et les personnes concernés ne constituent aujourd'hui un ensemble homogène. Cette fragmentation rend singulierement difficile l'appréhension des dynamiques à l'œuvre dans ces métiers et la définition de politiques publiques visant leur professionnalisation, alors qu'ils sont au cour des politiques en faveur de l'accès à la culture.

Disposer d'un état des lieux de l'ensemble des situations professionnelles rencontrées sous l'intitulé générique de «médiation culturelle», proposer une approche renouvelée de ces activités du point de vue de l'organisation du travail étaient les premiers objectifs de l'étude des métiers de la médiation culturelle. Elle met au jour un paradoxe : si les activités de médiation font l'objet d'un large consensus, elles demeurent aujourd'hui faiblement insérées au sein des organisations et institutions culturelles et elles sont généralement portées par des acteurs professionnels aux conditions d'emploi précaires, aux statuts et formes de reconnaissance incertaines.

Pareil constat doit sensibiliser les structures culturelles à la nécessité de mettre en cuvre des politiques de ressources humaines visant à structurer, accompagner et renouveler ces activités de médiation au regard de leurs enjeux actuels et futurs et compte tenu de leur fonction centrale pour les politiques des publics. L'équipe qui a réalisé cette analyse, en dépit de la disparition regrettée de l'un de ses membres, ouvre là un champ de réflexion et d'action particulièrement neuf et utile.
\end{abstract}

Philippe Chanterte
Visites guidées, ateliers, partenariats pédagogiques, rencontres et festivals artistiques, conférences... la médiation dans les lieux culturels est omniprésente et multiple. Scolaires, touristes, adultes en groupe ou individuels, seniors, abonnés en bénéficient. Pas un secteur, pas une institution, pas un lieu culturel, quelle que soit sa taille, ne semble aujourd'hui pouvoir l'ignorer. Du statut controversé de dispositif innovant il y a vingt ans, la médiation est devenue à présent une sorte d'évidence pour le champ culturel. Accompagnant ce mouvement, la littérature académique souligne les ambitions et le potentiel régénérant de la médiation pour répondre aux objectifs de démocratisation culturelle. Pour toutes ces raisons, la médiation culturelle fait figure de continent. Pourtant, un rapide examen des activités de médiation proposées par les lieux culturels la fait apparaître aussi comme une sorte de constellation, faite de pratiques hétérogènes, servant des intérêts divers et reposant sur des savoirs et des compétences variés. Dispersée, la médiation serait-elle davantage un archipel qu'un continent?

\footnotetext{
A Olivier,

sans qui ce texte et bien d'autres choses n'auraient pas pu voir le jour et qui nous manque beaucoup.

* Enseignants chercheurs au Centre de gestion scientifique de l'École des mines-Paris Tech.
} 


\section{Médiation culturelle : l'enjeu de la gestion des ressources humaines}

Cultural Promotion : The Human Resource Management Issue

Nicolas Aubouin, Frédéric Kletz et Olivier Lenay

Éditeur : Département des études, de la prospective et des statistiques

Lieu d'édition : Paris

Année d'édition : 2010

Date de mise en ligne : 8 juillet 2015

Collection : Culture études

ISBN électronique : 9782111398948

\section{Sbooks}

http://books.openedition.org

\section{Édition imprimée}

Date de publication : 1 mai 2010

Nombre de pages : 12

\section{Référence électronique}

AUBOUIN, Nicolas ; KLETZ, Frédéric ; et LENAY, Olivier. Médiation culturelle : l'enjeu de la gestion des ressources humaines. Nouvelle édition [en ligne]. Paris : Département des études, de la prospective et des statistiques, 2010 (généré le 25 avril 2021). Disponible sur Internet : <http://

books.openedition.org/deps/233>. ISBN : 9782111398948. 

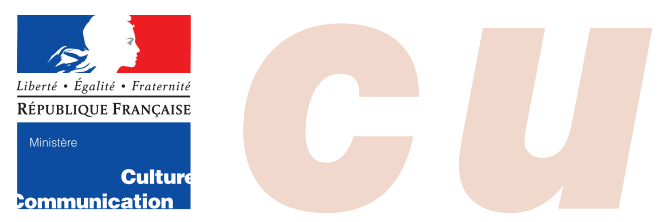

Secrétariat général

Service de la

coordination des

politiques culturelles

et de l'innovation

Département

des études,

de la prospective

et des statistiques

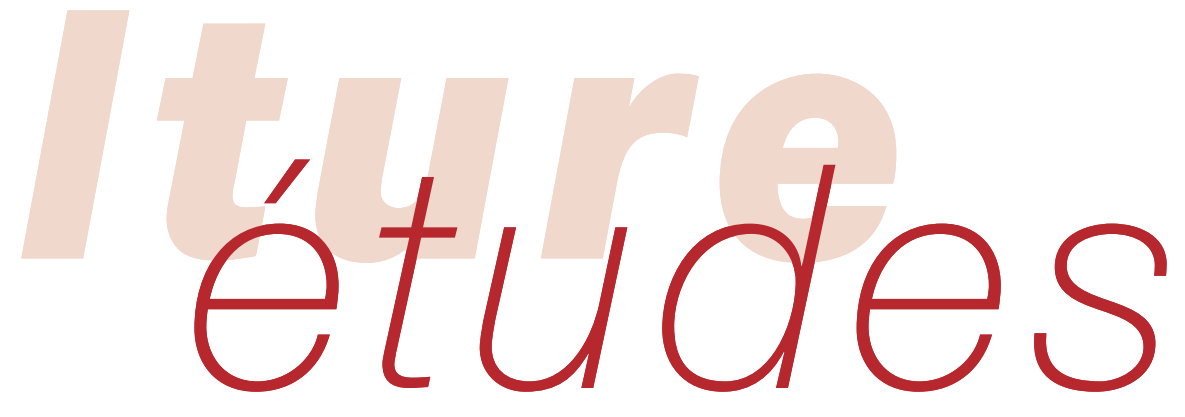

\title{
Médiation culturelle: l'enjeu de la gestion des ressources humaines
}

\author{
Nicolas Aubouin, Frédéric Kletz, Olivier Lenay*
}

\section{Cultural Promotion: The Human Resource Management Issue}

\section{Avant-propos}

Depuis plus de trente ans, les activités de médiation culturelle, qui visent à favoriser l'appropriation collective des différentes formes d'art et de patrimoine, ont connu un essor continu dans l'ensemble des domaines artistiques et culturels. Pour autant, ni ces activités, ni les emplois et les personnes concernés ne constituent aujourd'hui un ensemble homogène. Cette fragmentation rend singulièrement difficile l'appréhension des dynamiques à l'ouvre dans ces métiers et la définition de politiques publiques visant leur professionnalisation, alors qu'ils sont au cour des politiques en faveur de l'accès à la culture.

Disposer d'un état des lieux de l'ensemble des situations professionnelles rencontrées sous l'intitulé générique de "médiation culturelle", proposer une approche renouvelée de ces activités du point de vue de l'organisation du travail étaient les premiers objectifs de l'étude des métiers de la médiation culturelle. Elle met au jour un paradoxe : si les activités de médiation font l'objet d'un large consensus, elles demeurent aujourd'hui faiblement insérées au sein des organisations et institutions culturelles et elles sont généralement portées par des acteurs professionnels aux conditions d'emploi précaires, aux statuts et formes de reconnaissance incertaines.

Pareil constat doit sensibiliser les structures culturelles à la nécessité de mettre en ouvre des politiques de ressources humaines visant à structurer, accompagner et renouveler ces activités de médiation au regard de leurs enjeux actuels et futurs et compte tenu de leur fonction centrale pour les politiques des publics. L'équipe qui a réalisé cette analyse, en dépit de la disparition regrettée de l'un de ses membres, ouvre là un champ de réflexion et d'action particulièrement neuf et utile.

Philippe Chantepie
Visites guidées, ateliers, partenariats pédagogiques, rencontres et festivals artistiques, conférences... la médiation dans les lieux culturels est omniprésente et multiple. Scolaires, touristes, adultes en groupe ou individuels, seniors, abonnés en bénéficient. Pas un secteur, pas une institution, pas un lieu culturel, quelle que soit sa taille, ne semble aujourd'hui pouvoir l'ignorer. Du statut controversé de dispositif innovant il y a vingt ans, la médiation est devenue à présent une sorte d'évidence pour le champ culturel. Accompagnant ce mouvement, la littérature académique souligne les ambitions et le potentiel régénérant de la médiation pour répondre aux objectifs de démocratisation culturelle. Pour toutes ces raisons, la médiation culturelle fait figure de continent. Pourtant, un rapide examen des activités de médiation proposées par les lieux culturels la fait apparaître aussi comme une sorte de constellation, faite de pratiques hétérogènes, servant des intérêts divers et reposant sur des savoirs et des compétences variés. Dispersée, la médiation serait-elle davantage un archipel qu'un continent?

\section{À Olivier,}

sans qui ce texte et bien d'autres choses n'auraient pas pu voir le jour et qui nous manque beaucoup.

\footnotetext{
* Enseignants chercheurs au Centre de gestion scientifique de l'École des mines-Paris Tech.
} 
Que dire des personnes - les médiateurs culturels - qui mettent en œuvre l'activité de médiation? Quel paysage de la médiation cette catégorie d'acteurs, née de la conjonction d'initiatives militantes en lutte contre les fatalités de la reproduction culturelle et de politiques publiques de soutien à l'emploi, compose-t-elle ? Qui sont les médiateurs qui travaillent dans les lieux culturels? En quoi consiste leur rôle et à quels problèmes font-ils face dans l'exercice de leurs fonctions?

L'intérêt de cette étude ${ }^{1}$ est d'aborder d'un point de vue dynamique les contenus de l'activité de médiation, les compétences attachées, les conditions d'emploi (accès à l'emploi, statuts, rémunération, temps de travail...), la définition des postes de travail ainsi que les relations au sein des organisations, selon les structures et leur politique en termes de gestion des ressources humaines.

Enfin, dans un contexte de développement, depuis plus de trente ans, des activités de médiation, la question d'une éventuelle spécificité sectorielle se pose : certains secteurs (musées, patrimoine, cinéma, arts visuels...) sont-ils parvenus à offrir aux populations de médiateurs des conditions de réalisation de leur activité plus favorables, notamment en termes de statut et de déroulement de carrière?

\section{La médiation au regard d'évolutions majeures du travail et de l'emploi}

Au-delà de la seule réactualisation des connaissances, la question de la médiation peut contribuer à éclairer plusieurs enjeux actuels. D'une part, elle appartient à une catégorie d'activités dans laquelle le travail n'est pas stabilisé et échappe le plus souvent aux cadres statutaires de la sphère publique. Du même coup, les contours professionnels de l'activité sont mal définis, les acteurs, à l'image professionnelle peu claire, ne bénéficient pas d'un statut stable et effectuent des allers-retours entre différentes activités (médiation, pratiques artistiques, formation...). En abordant la question de la précarité, l'étude peut nourrir la réflexion sur la difficile structuration professionnelle de certaines activités culturelles.

D'autre part, la crise économique et son impact social posent avec acuité la question de l'action publique, notamment en matière de lutte contre le chômage : quels emplois aidés et dispositifs d'accompagnement mettre en place ? Le secteur culturel - et notamment la médiation (dont l'histoire est étroitement associée à celle de ces dispositifs) est particulièrement concerné et fait l'objet d'un intérêt particulier de la part des politiques publiques.

\section{L'apport des sciences de gestion à la sociologie des professions}

Plus peut-être que pour d'autres activités et en raison de son projet d'établir des pratiques professionnelles à l'interface de différents mondes, le choix a été fait de rendre compte de la dimension à la fois coopérative et organisée de cette activité, mais aussi du mouvement de professionnalisation de la médiation culturelle. Il s'agit donc de relier l'activité de médiation aux organisations (institutions, réseaux, collectifs, etc.) dans lesquelles elle est mise en œuvre, d'analyser comment les médiateurs s'inscrivent dans un contexte qui leur confère un espace de déploiement : attributs, compétences, coordination avec les autres activités. C'est cet espace, dans sa topographie, qui est désigné par le concept de configuration professionnelle de la médiation (CPM).

Enfin, l'approche vise à concilier deux perspectives théoriques (la large distribution de la fonction de médiation au sein de chaque institution culturelle, au-delà d'un métier unique ou spécifique d'une part ; le métier de médiateur d'autre part), en y introduisant un volet organisationnel: dans quelles configurations professionnelles les médiateurs, en tant que métier ou fonction, sont-ils amenés à intervenir? Au sein de quelle combinaison de ressources cette activité est-elle intégrée ? Quels sont les autres acteurs avec lesquels ils ont à interagir? Dans quelles catégories de compétences les représente-t-on?

Enfin, l'analyse des compétences des médiateurs a été appréhendée au travers de leur déploiement au sein de configurations professionnelles, qui les renouvellent ou les appauvrissent. Plus que pour tout autre population, les compétences ne peuvent être abordées comme un stock mais au contraire comme un processus.

\section{L'ÉVIDENCE DE LA MÉDIATION}

\section{Une médiation généralisée mais multiforme}

Les quelque cinquante lieux culturels observés, quels que soient leur taille, leur statut et le domaine culturel qu'ils représentent, proposent tous des activités de médiation. Ainsi, la médiation est devenue une évidence pour toute institution : théâtres, musées nationaux, bibliothèques municipales, opéras, services d'archives, salles de cinéma, centres d'art contemporain, monuments historiques, orchestres... proposent une large gamme d'actions dites de médiation, que certaines institutions déclarent comme le cœur de leur mission.

La partie la plus visible de telles activités concerne l'offre d'activités de médiation proposée au grand public: visites, conférences, ateliers, etc. On observe aussi le développement d'offres plus ciblées, en relation avec une typologie des publics visés (scolaires, groupes constitués, érudits, etc.) ainsi que la conception de projets sur mesure, en réponse à la demande de publics particuliers (milieux pénitentiaire et hospitalier, classes de l'Éducation nationale...).

En dépit de cette banalisation de la médiation, il importe de souligner - c'est un paradoxe - que la terminologie de médiateur ou de médiation est rarement utilisée, et même souvent volontairement évitée au profit d'expressions comme : action culturelle, action pédagogique, relation aux publics, action territoriale, animation scientifique, action musicale, etc. La médiation apparaît comme un mot valise renvoyant à une grande hétérogénéité d'activités.

1. Nicolas Aubouin, Frédéric KletZ, Olivier Lenay (Centre de gestion scientifique de l'École des mines-Paris Tech), Entre continent et archipel, les configurations professionnelles de la médiation, Paris, DEPS, Ministère de la Culture et de la Communication, coll. «Document de travail», 2009-1, novembre 2009. 
L'action pédagogique occupe une place centrale dans le champ de la médiation. Quel que soit le lieu, l'activité de médiation est en grande partie dédiée aux publics scolaires (des maternelles aux étudiants du supérieur, avec une priorité donnée aux primaires). Cela s'explique à la fois par la présence de réseaux institutionnalisés, par l'existence de préoccupations éducatives nationales et locales, mais également parce que le public scolaire constitue un levier pour la démocratisation culturelle, qu'il est captif, qu'on se représente ses attentes et enfin qu'il correspond à des effectifs importants. Cette orientation massive de la médiation a des conséquences sur les profils des médiateurs qui en ont la charge.

\section{Une inscription dans le temps plus ou moins récente selon les secteurs}

La généralisation de la médiation ne s'est pas opérée au même moment, selon les secteurs et selon les territoires : si le secteur des musées s'est depuis longtemps doté d'équipes structurées en charge des questions de médiation, le secteur du spectacle vivant ou celui des archives y sont venus plus récemment, bien que les questions d'accueil du public ou de transmission s'y posent depuis longtemps. Enfin, au-delà d'éventuelles variations sectorielles, le développement des activités de médiation s'est souvent opéré à la faveur d'opportunités au plan local (politiques d'aide à l'emploi public, subventions, soutien des collectivités territoriales...).

\section{Une généralisation favorisée par les pouvoirs publics}

Alors que l'État a contribué largement depuis plus de vingt ans à l'accroissement des actions de médiation dans les grandes institutions comme dans les petites associations culturelles, par des financements ou des dispositifs invitant au développement des actions éducatives, les collectivités territoriales jouent depuis quelques années un rôle de plus en plus central, à travers la multiplication de dispositifs locaux d'incitation et de gestion des activités de médiation, mais aussi de nouvelles organisations des services culturels à caractère transversal.

La politique des collectivités s'est construite autour de deux logiques : le territoire et les publics. Les collectivités ont en effet cherché à concilier la valorisation par la culture de certaines zones géographiques (zones rurales, quartiers sensibles...) et les enjeux de démocratisation culturelle (augmentation et élargissement des publics). Il apparaît toutefois certaines limites à cette politique. Ainsi, il n'existe toujours pas de cadre d'emploi spécifique de médiateur dans la fonction publique territoriale. Autrement dit, une collectivité qui souhaite embaucher un médiateur pour son musée ou son théâtre est contrainte soit de puiser dans le vivier des lauréats aux concours ouverts pour un autre cadre d'emploi, type «attaché de conservation » ou «agent qualifié du patrimoine », soit de recruter par voie contractuelle (CDI, CDD, vacataires...). Par ailleurs, les lauréats de concours ayant choisi l'option «médiation » aux concours n'ont aucune garantie d'être affectés à un poste correspondant à cette option.

Les pouvoirs publics interviennent aussi par l'intermédiaire de l'expertise : en ce qui concerne l'État, par le biais, notamment, des conseillers sectoriels des directions régionales des affaires culturelles; en ce qui concerne les collectivités, par le biais de leurs personnels spécialisés. Bien qu'ils ne se reconnaissent pas comme médiateurs, ces agents jouent un rôle essentiel dans le développement de projets de médiation, soit par le conseil, soit par la gestion directe ou par la contribution au financement des structures.

\section{UNE APPROCHE NOUVELLE : LA CONFIGURATION PROFESSIONNELLE}

Comme les diverses dénominations attachées aux postes de travail le suggèrent (chargé d'action culturelle, guide conférencier, animateur, etc.), le terme de médiateur culturel est une fiction qui renvoie davantage à l'importance prise au plan théorique par le concept de médiation dans le champ culturel, ainsi qu'à l'usage qu'en ont fait certaines politiques publiques de soutien à l'emploi, plutôt qu'à une réalité. Trop générique, le terme de «médiateur» appelle une représentation plus précise du paysage de la médiation. Deux axes structurants se dégagent, qui permettent de distinguer simultanément activités et compétences de médiation.

\section{La division du travail}

Le premier axe s'organise autour de deux pôles, à l'extrémité desquels se trouvent :

- des concepteurs producteurs de dispositifs de médiation ${ }^{2}$ : diversement nommés (chargés d'action culturelle, chargés d'action territoriale, chargés de médiation, chargés de projet, responsables des publics, etc.), ils doivent concevoir la réalisation d'une action de médiation face au public. Ils assurent en général l'ensemble de la production de ces modules, sous l'angle organisationnel et logistique : contact avec les artistes et les compagnies, avec des partenaires extérieurs pour les activités hors les murs, élaboration des contrats, rémunération des équipes artistiques et des techniciens... Leur implication dans l'élaboration du contenu de la prestation est variable : parfois nulle lorsqu'ils achètent des produits clés en main, elle peut être importante dans certains cas, lorsque le producteur monte lui-même une exposition ou lorsqu'il élabore les scénarios de l'atelier ou de la visite ;

\footnotetext{
2. Par dispositif de médiation, on entend la conception du cadre dans lequel les interactions de médiation se déroulent (y compris leur propre rôle à l'intérieur de cet espace, ce qui explique qu'on les identifie eux-mêmes comme acteurs de la médiation). Concevoir un tel cadre peut nécessiter des compétences différentes selon les cas, mais impose que soient spécifiés, de manière plus ou moins explicite : les publics visés, le cadre spatio-temporel dans lequel la médiation se déroule, le type de contenu qui doit être transmis, utilisé ou produit, etc. Cette conception peut être le produit d'une démarche collective effectuée en interne ou bien d'une démarche menée en partenariat (collectivités locales, structures associatives, autres acteurs publics...).
} 
- des animateurs face au public : chargés de la réalisation des activités de médiation face au public, quels que soient le format, le contenu, le mode opératoire. Le terme d'animateur, même s'il rappelle l'animation socioculturelle et reste connoté dans le milieu de la médiation, permet de décrire l'activité de mise en œuvre des activités de médiation face au public.

Dans cette population se trouvent deux catégories d'intervenants :

- des artistes qui effectuent une prestation artistique à finalité de médiation culturelle: ateliers de pratique musicale, de pratique théâtrale, d'arts plastiques, démonstrations, atelier chorégraphique, conférence d'explication de sa proposition artistique (l'artiste exposé dans un centre d'art, le metteur en scène...). Ces ateliers et conférences peuvent se faire dans l'enceinte de la structure ou hors les murs (prison, hôpital, école, salle de quartier...) ;

- des non-artistes : conférenciers, guides conférenciers, animateurs d'ateliers pédagogiques (notamment pour le jeune public). Ces modules ont lieu généralement dans les murs de l'institution culturelle.

En opposant les animateurs aux concepteurs producteurs, il ne s'agit pas simplement de reprendre la division classique entre conception et exécution. Les animateurs réalisent, pour la plupart d'entre eux, une partie de la conception des activités de médiation, non pas des dispositifs de médiation, dont la création relève du concepteur, mais plutôt les contenus et les modes opératoires élémentaires de la médiation. Ils peuvent donc être relativement autonomes tout en n'étant pas en charge de la conception de l'économie générale de leur intervention.

Selon la taille des structures, une telle division du travail est plus ou moins marquée. Dans les petites structures, les profils sont plus polyvalents, assurant à la fois la conception des actions de médiation et leur mise en œuvre face au public. On les appelle les concepteurs animateurs. En revanche, lorsque les structures accueillent un nombre important de visiteurs, on assiste à une spécialisation des activités.

La division du travail peut aussi résulter d'une stratégie : certains lieux - même de grande taille - estiment qu'il est nécessaire de maintenir un lien entre les activités de conception et de mise en œuvre et s'attachent à promouvoir la polyvalence. L'inverse existe également, certaines structures souhaitant accentuer la professionnalisation des activités et estimant qu'elles relèvent de deux registres professionnels différents.

Ces choix stratégiques sont liés à l'activité propre à chaque structure et dépendent de la nature des publics accueillis, des contenus culturels proposés, des partenaires externes, mais relèvent aussi de la séparation des compétences et des activités qui s'opère selon un deuxième axe.

\section{La spécialisation : contenu ou public}

Moins spontanément mobilisé par les acteurs, et sans doute aussi plus sujet à controverse, ce second axe structure le paysage des formes professionnelles de la médiation en distinguant, d'une part, des acteurs dont l'intervention est plutôt orientée vers les contenus (histoire de l'art, pratique artistique, histoire des sciences et pratiques scientifiques, etc.) et, d'autre part, des acteurs dont l'intervention est orientée vers les publics. Si certains, par leur formation initiale ou par leur pratique professionnelle quotidienne, développent et articulent des compétences relevant de ces deux pôles, dans la plupart des cas, les médiateurs se caractérisent soit par une dominante «contenus » soit par une dominante «publics ».

La dominante de contenus s'observe lorsque les connaissances, les compétences et les activités sont de nature disciplinaire (disciplines artistiques ou scientifiques). À l'opposé, dans la dominante publics, la maîtrise porte essentiellement sur la nature, la typologie et les comportements des publics. On trouve par exemple dans cette seconde catégorie des compétences d'ingénierie de partenariats adaptés à un public particulier, des connaissances relatives aux pratiques de consommation des publics, des spécialistes du public scolaire et des partenariats avec l'Éducation nationale.

Les deux axes permettent de disposer d'un espace de représentation à deux dimensions (axe horizontal : conception/face-à-face avec le public, et axe vertical : dominante contenus/dominante publics). On appelle alors configuration professionnelle de médiation (CPM) l'espace de positionnement des différents acteurs de médiation selon leurs activités et compétences repérées par les axes conception/face-à-face avec le public d'une part, et contenu/public de l'autre, mais aussi les relations qui les lient au sein de cet espace. Le choix de cette représentation vise à montrer que la médiation est réalisée par un collectif composé de différents professionnels, à l'intérieur et à l'extérieur des lieux culturels, et que chacun possède ses propres traits de professionnalisation (profession, métier ou fonction). Un premier exemple permet de saisir ces différentes dimensions.

L'exemple du service d'archives (encadré p. 6) illustre les possibilités d'analyse de l'activité de médiation qu'offre le concept de CPM : couverture de l'espace des compétences nécessaires, adéquation aux types de publics, modes de coordination entre les différents acteurs, etc.

La modélisation permet, en ne retenant que la représentation des acteurs de la médiation et des relations qu'ils entretiennent, de repérer des configurations types à partir des différentes situations rencontrées.

\section{Six configurations types}

Si l'on restreint la représentation aux acteurs dédiés à la médiation, on dispose alors d'une représentation par construction partielle qui concerne le cœur de métier de la médiation. Ainsi simplifiée, une CPM type permet d'identifier le choix qui a présidé à la conception d'une organisation de la médiation et de repérer les formes génériques de spécialisation. Elle permet aussi, en comparant une CPM type à la CPM complète qui décrit l'organisation d'un lieu, d'identifier le degré de couverture de l'espace des activités et des compétences et la nécessité de s'assurer le concours d'autres types d'acteurs.

Six CPM types différentes peuvent être modélisées, allant d'une situation où des médiateurs assurent euxmêmes toutes les tâches (de la conception des dispositifs 


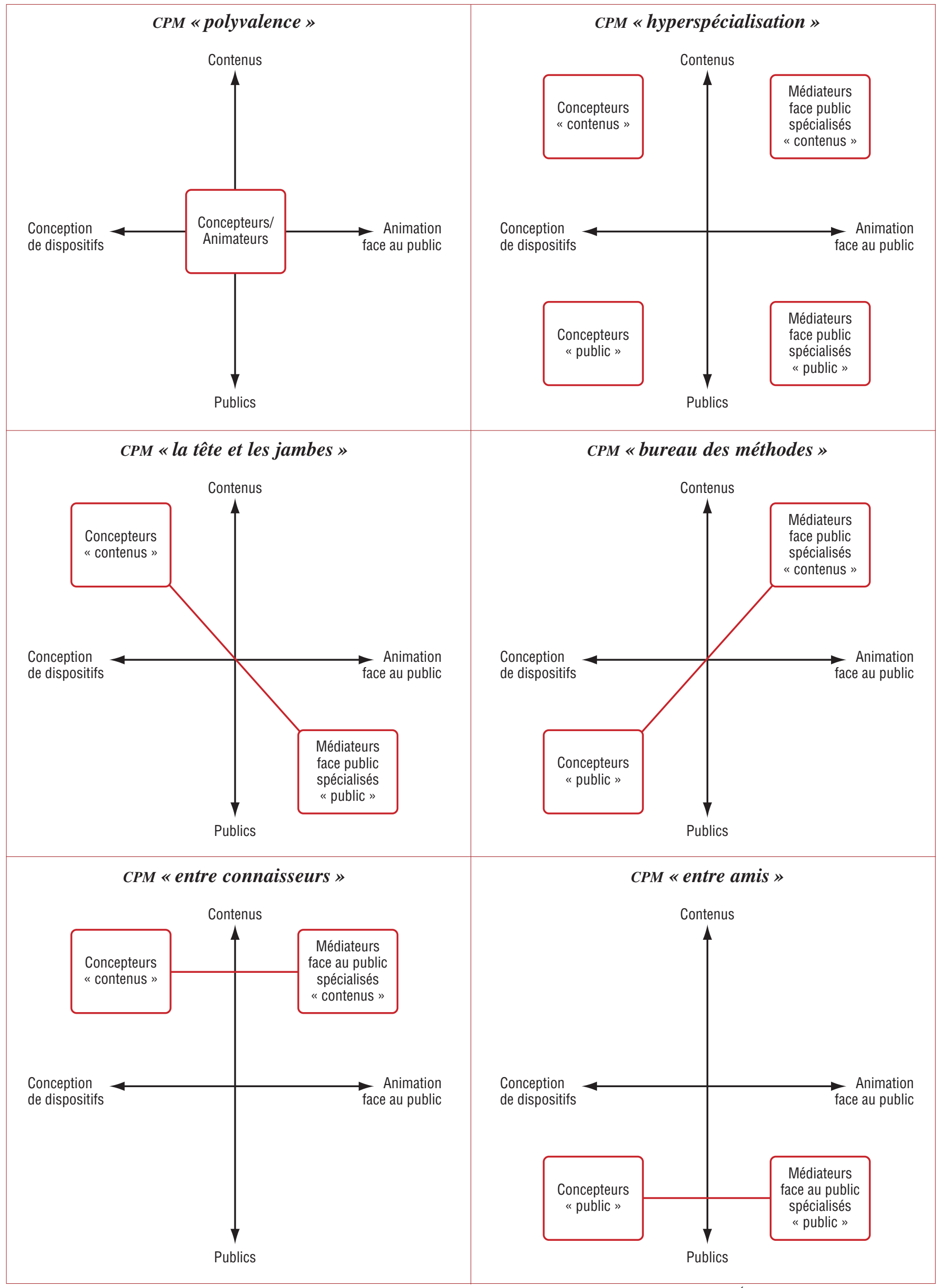




\section{La médiation dans un service d'archives municipales : approche sous forme de CPM}

Ce service d'archives municipales, rattaché à la direction des affaires culturelles est considéré comme un service d'archives important (45 agents, $15 \mathrm{~km}$ d'archives, dont $80 \%$ de fonds d'archives municipales).

Son organisation repose sur un découpage en deux pôles opérationnels (logistique et administration) et trois pôles fonctionnels : gestion des fonds, salle de lecture, action culturelle et pédagogique. C'est dans le dernier pôle que sont menées les actions de médiation.

Le service organise en moyenne une exposition par an, d'une durée d'environ 4 à 6 mois. Sa conception est assurée par un permanent employé à temps plein à cette fonction et qualifié de «chargé des expositions ", avec l'aide d'un ou plusieurs partenaires extérieurs, tandis que son animation est assurée par un vacataire, recruté sur un contrat qui couvre la durée de l'exposition, pour un volume horaire hebdomadaire de 25 heures, généralement étudiant des filières tourisme ou valorisation du patrimoine culturel. Les activités proposées sont variées : visites guidées, projections, lectures d'archives par des comédiens, conférences et débats, etc. Certaines de ces activités ont lieu hors les murs, parfois en association avec d'autres types d'animateurs en face-à-face avec le public (exemple d'une visite d'un cimetière de la ville dans le cadre d'une exposition sur la généalogie).

Du côté des actions pédagogiques, le service est composé d'un permanent (le responsable des actions pédagogiques) et, selon les périodes, d'un certain nombre d'animateurs en face-à-face avec le public, appelés médiateurs, recrutés sur des emplois aidés. Le responsable du service est titulaire d'un DESS d'archivistique.

Tandis que les publics accueillis dans les expositions temporaires sont principalement des publics récepteurs, qu'il s'agisse de publics spécialisés, de scolaires ou de grand public, ceux des actions pédagogiques sont plutôt concepteurs, dans le cadre d'une logique de projet spécifiquement développée pour et avec eux. II s'agit dans ce cas d'accompagner les projets de partenaires (Éducation nationale, milieu pénitentiaire, monde associatif, etc.) dans une logique de partage des compétences. Une partie des activités de médiation consiste à former les acteurs de ces projets aux archives (enseignants, éducateurs) afin qu'ils assurent eux-mêmes certaines actions de médiation, en relation avec leurs propres projets (travail avec une classe sur l'histoire de son établissement, avec une prison sur les plans, etc.). Ce travail partenarial est effectué par le responsable du service mais aussi par les médiateurs. Ces deux catégories d'acteurs peuvent en outre assurer eux-mêmes des activités en face-à-face avec le public.

La configuration professionnelle de médiation de ce service d'archives peut être modélisée ainsi :

\section{Graphique 2 - Modélisation de la médiation dans un service d'archives municipales}

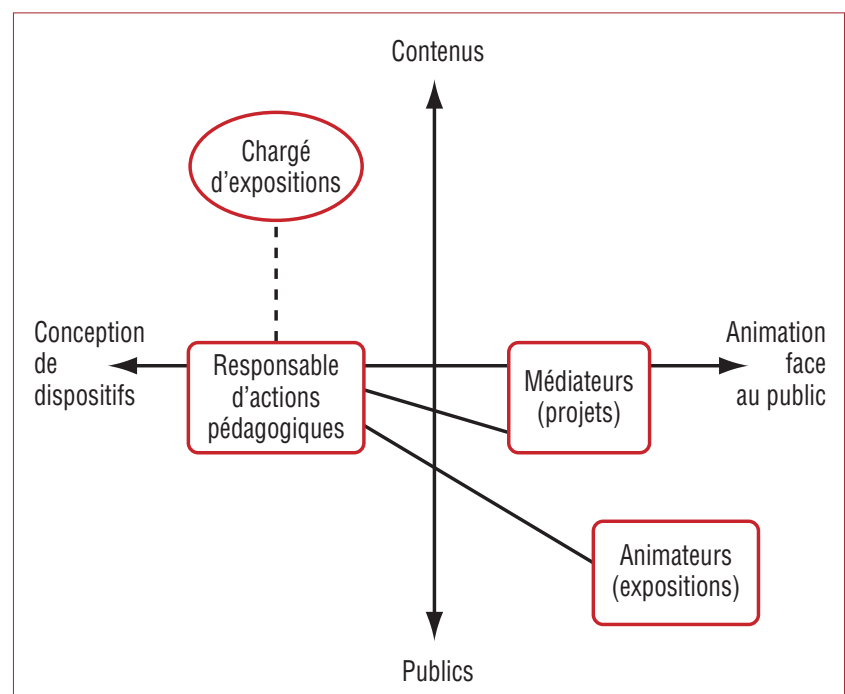

Note de lecture : les conventions de représentation sont les suivantes - les acteurs dédiés à la médiation sont représentés par un rectangle, ceux qui ont une fonction de médiation par un rond ;

- leurs relations sont représentées par des traits continus lorsqu'il s'agit de relations hiérarchiques, des traits discontinus pour des relations d'une autre nature (ici de coopération au sein d'un même service).

Source : Médiation culturelle, École des mines-ParisTech/DEPS, 2010 de médiation jusqu'à leur mise en œuvre, tout en intégrant des compétences de contenus et de publics) jusqu'à la situation opposée où chaque portion de l'espace des activités et des compétences est assurée par un acteur dédié ${ }^{3}$ (graphique 2). Entre ces cas, extrêmes, des configurations qui occupent une couverture partielle de l'espace des activités et des compétences sont identifiées.

- La CPM «polyvalence » illustre l'hypothèse souvent formulée, implicite ou non, selon laquelle les profils de médiateurs se doivent de détenir un éventail de compétences très large, allant de compétences sur les contenus culturels concernés jusqu'à des compétences orientées vers les publics. Lorsque, de surcroît, les médiateurs ont à la fois pour mission de concevoir des dispositifs de médiation et de les mettre concrètement en œuvre face au public, on se trouve alors en présence d'une figure d'acteur réellement polyvalente, d'un médiateur chef d'orchestre tenant tous les bouts de l'activité et des compétences de médiation.
- La CPM «hyperspécialisation» renvoie à la situation inverse. Une telle configuration, compte tenu du nombre d'acteurs envisagés (et on ne considère ici que des acteurs dédiés à la médiation) et de l'organisation qu'il convient de mettre en place pour coordonner ces différents acteurs, est réservée à des structures culturelles déjà très développées.

- Parmi les CPM obliques, la CPM «bureau des méthodes » est la plus souvent rencontrée. Elle implique une division du travail assez hiérarchisée entre concepteurs et médiateurs face au public, des généralistes d'un côté, des spécialistes des publics de l'autre. Cette configuration est viable notamment pour des structures de taille relativement importante, qui disposent d'un service des publics ou de responsables dédiés à l'activité de conception de la médiation. Elle implique aussi des relations régulières entre concepteurs de projet de médiation et acteurs responsables des contenus (conservateurs des musées par exemple),

3. Les CPM obliques se caractérisent par une distinction stricte entre activités de conception et activités d'animation, spécialisation des activités vers les contenus ou vers les publics. Les CPM plates caractérisent des activités de médiation spécialisées, soit vers les contenus, soit vers les publics. 
d'autant plus nécessaires si les relations entre médiateurs concepteur et face au public sont distendues (dans les cas d'externalisation de l'activité face au public notamment). - La CPM « la tête et les jambes » se caractérise par l'extériorité des concepteurs de projet (appartenant par exemple au Centre national du cinéma et de l'image animée dans le secteur du cinéma ou à une collectivité territoriale coordonnant une politique). Les médiateurs face au public font appel aux outils développés par ces concepteurs extérieurs pour réaliser leurs activités.

- Parmi les CPM plates, la première, «entre connaisseurs », est fréquente dans le secteur du patrimoine. En effet, historiquement les seuls intervenants dans ce secteur étaient les guides conférenciers, aujourd'hui encore recrutés principalement pour leurs compétences en histoire de l'art. Leurs fonctions et leur autonomie les conduisaient à couvrir le spectre allant de la conception jusqu'à la réalisation d'actions de médiation, sans véritable dispositif de capitalisation des connaissances produites. L'arrivée des animateurs du patrimoine qui, comme leur nom ne l'indique pas, sont principalement des concepteurs de dispositifs de médiation, est venue introduire une concurrence du côté de la conception. Cette configuration peut être viable dans les cas où le besoin d'une ingénierie en direction des publics est relativement limité (cas par exemple des monuments historiques où l'itinéraire de visite, s'il ne standardise pas le discours, le cadre fortement).

- La seconde CPM plate, «entre amis », est pertinente dans le cas où les publics sont avant tout en demande de médiation pour satisfaire des objectifs extérieurs au champ culturel. Un groupe de scolaires qui mobilise un équipement culturel en raison de son lien avec des objectifs pédagogiques en est l'exemple typique. Cette configuration suppose que les contenus culturels soient définis par ailleurs, appropriables par les concepteurs et face au public à dominante publics. Il y a, dans ce cas comme dans le précédent, indépendance entre une ingénierie des contenus et une ingénierie des publics.

\section{Une médiation sectorielle ?}

La question des spécificités sectorielles de la médiation peut être étudiée à partir de ces six configurations types. Contrairement à ce que soulignent certains acteurs, la médiation semble davantage structurée par des considérations spécifiques à chaque lieu (quel type de public, quel projet et quelles activités de médiation, quels partenariats, etc.) que par une quelconque spécificité sectorielle et il est difficile d'identifier un certain type de configuration à un secteur. En revanche, les configurations se retrouvent dans différents secteurs et différents lieux. Certains lieux ont en outre migré d'une configuration à une autre (cas d'une institution passant de la polyvalence à l'hyperspécialisation ou d'une autre qui a progressivement accentué la division du travail entre concepteurs orientés publics et animateurs face au public orientés vers les contenus).

La polyvalence n'est pas une conséquence de la taille des structures, ni du type de secteur. A priori, celle-ci aurait pu être réservée à des lieux de petite taille ou à des cas où la délégation de tâches semble difficile à mettre en œuvre. En réalité, elle concerne des lieux de taille et de secteur variables et elle constitue par conséquent davantage un choix stratégique. Elle n'est pertinente que dans le cas d'une action sur mesure pour les publics, donc peu stable (le développement important des publics scolaires, qui nécessite des acteurs dédiés, donne souvent le signal de l'explosion du modèle).

La CPM «bureau des méthodes » est la plus fréquemment rencontrée. Une première explication de la forte représentation de cette configuration est donc de type généalogique : elle témoigne de l'histoire centrale de la médiation dans laquelle la relation au public était première mais peu assujettie aux questions de la démocratisation culturelle. Au-delà, elle est celle qui permet le mieux d'organiser la sous-traitance de l'animation face au public ; elle est la plus en phase avec un dogme de l'animation face au public avant tout culturelle (par opposition ou rejet d'une animation socioculturelle), et permet de traiter une gamme assez large de publics.

\section{Graphique 3 - Répartition des CPM dans les structures enquêtées}



Source : Médiation culturelle, École des mines-ParisTech/DEPS, 2010 
UNE ANALYSE DE LA SITUATION EN TERMES DE RESSOURCES HUMAINES

L'explicitation des configurations professionnelles révèle l'existence de facteurs de différenciation de la population des médiateurs :

- les compétences : profils de médiateurs des institutions culturelles, liens avec les configurations professionnelles, types de compétences développées ;

- l'organisation : place des activités de médiation dans l'organisation; conditions de travail des médiateurs, outils de gestion des lieux culturels;

- la professionnalisation: degré de reconnaissance de ces acteurs, facteurs d'évolution et dynamiques de professionnalisation, trajectoires professionnelles des médiateurs.

\section{Compétences transversales partagées ou spécifiques?}

L'absence de reconnaissance des compétences, exprimée par les médiateurs eux-mêmes, est le premier élément à souligner. Non pas que celles-ci soient niées, mais plutôt qu'elles ne sont nulle part explicitées, en particulier dans les référentiels statutaires ou les répertoires d'emplois.
Ce qui, pour des activités encore en émergence dans certains lieux, au positionnement toujours fragile, peut amplifier le sentiment d'absence de légitimité. Faute d'une formalisation par les institutions culturelles employeuses, un travail d'exploration et d'identification des différentes compétences mobilisées par les populations des médiateurs a été réalisé. Alors que la distinction entre les compétences, savoirs et savoir-faire des médiateurs concepteurs d'une part et ceux en situation de face-à-face avec le public d'autre part, paraît relativement aisée, la ligne de partage d'être mieux précisée. Le travail de médiation, dans ses différentes configurations professionnelles, fait appel à des gammes de compétences et des champs d'expertise relativement importants. À des compétences transversales à l'ensemble des configurations professionnelles s'ajoutent des compétences spécifiques, qui spécialisent les métiers de la médiation.

Du côté des animateurs face au public, qu'ils soient orientés vers les contenus ou vers les publics, les compétences (connaissances, savoir-faire) partagées ont évidemment d'abord trait à la prise en charge des publics (capacité d'adaptation et gestion de l'interactivité), mais deux autres composantes sont mises en avant par les médiateurs, bien que souvent occultées au sein même de leur organisation : leur capacité à prendre appui sur les connaissances

\section{La médiation au cœur du projet de l'établissement}

\section{La politique de ressources humaines de médiation d'un musée d'art: une tentative d'articulation des activités, compétences, formations des médiateurs}

L'activité de médiation au sein de ce musée était au cœur du projet artistique de la première direction du lieu. Sa politique de médiation reposait essentiellement sur la mise en place, dans les lieux d'exposition, d'une équipe de médiateurs polyvalents (conseil, accueil, visite, caisse...) très autonomes. Elle s'est traduite par le recrutement de 12 médiateurs en emplois-jeunes, aux profils universitaires de haut niveau (doctorat pour certains). Les médiateurs se trouvaient dans les espaces, à la disposition des visiteurs, sans qu'il soit nécessaire de recourir à un quelconque dispositif préétabli : une médiation libre, ouverte, spontanée. Puis, assez vite, l'expérience a nécessité quelques réaménagements, d'abord légers (création de supports de médiation pour faciliter la prise de contact du public), puis plus importants, suite à des départs massifs au bout d'une année d'exercice. II fut alors fait appel à de nouveaux profils, de niveau bac +3 à bac +5 , issus de filières universitaires (histoire de l'art, architecture, cinéma, philosophie) ou de l'enseignement supérieur artistique.

Fondamentalement pourtant, les principes sur lesquels reposait le fonctionnement de la médiation restaient les mêmes, à savoir une perméabilité entre fonctions d'animation et de conception. Ces principes entraient d'ailleurs en résonance avec le modèle retenu à l'échelle du musée, modèle associatif, par opposition au modèle de l'institution, caractérisé à la fois par une forte polyvalence et une large liberté au plan de la gestion des équipes, et par une relative autonomie dans les rapports aux tutelles (à l'origine, le financement public ne représente que $50 \%$ du budget global).

Ce mode de fonctionnement a permis à différents médiateurs en face-à-face avec le public d'être forces de proposition pour la mise en place d'ateliers, d'événements, et de porter progressivement, au sein du service d'action culturelle, la conception des dispositifs de médiation, sous la forme d'ateliers pour enfants, de conférences et d'expositions hors les murs, de modules de visites en partenariat avec d'autres lieux de pratiques artistiques contemporaines.
L'exemple des ateliers pour enfants est révélateur du rôle joué par les médiateurs dans la mise en place d'un dispositif original de médiation. Le concepteur de ces ateliers, arrivé avec la seconde vague des médiateurs, a initié une activité dédiée au jeune public à travers des ateliers qui, au départ, avaient lieu dans les lieux d'exposition autour d'un " chariot nomade ». Ces ateliers se déplaçaient dans l'espace d'exposition et se déroulaient en trois temps : d'abord une visite de l'exposition, ensuite une réalisation plastique des enfants dans le cadre de l'atelier, enfin un accrochage des travaux des enfants à côté des œuvres des artistes. Le succès public et médiatique a alors permis le développement de cette activité : spécialisation d'un médiateur sur cette activité et recrutement de nombreux stagiaires.

À partir de 2006 et à la faveur d'un changement de direction, une nouvelle politique de médiation est mise en œuvre et institue un bureau des médiateurs (lieu d'information clairement identifié). Ce dispositif transforme l'activité des médiateurs : développement d'une activité de documentaliste (achat, classement, constitution d'archives), réduction de la polyvalence initiale des médiateurs, attachés essentiellement à des fonctions de visites et de conseils aux publics. Cette politique s'est ainsi accompagnée d'une réduction progressive du nombre de médiateurs. Certains postes de médiateurs ont été progressivement remplacés par des postes de caissiers et d'agents de sécurité.

L'équipe des médiateurs connaît un fort taux de remplacement dû soit au départ des personnes occupant un poste de médiateur, soit à l'évolution vers des postes de conception au sein de la structure. La forte rotation semble aussi liée à la nature de l'activité d'animation des espaces, qui exige une forte réactivité des équipes, ainsi qu'une certaine proximité avec le jeune public.

Ainsi, ce musée a progressivement quitté la configuration «polyvalence » pour entrer dans celle de l'« hyperspécialisation ». 
et les regards des publics pour en faire des ressources communes et des objets de dialogue avec les œuvres, mais aussi leur capacité à faire bénéficier l'institution de leurs connaissances des publics, acquises lors de leurs interventions. La médiation perd ainsi son caractère classiquement descendant pour, dans un cas, devenir davantage horizontale, et dans l'autre cas, ascendante. C'est l'une des principales difficultés rencontrées par cette population : faire reconnaître les acquis de l'expérience, au-delà des connaissances et des savoir-faire découlant d'une formation.

Du côté des concepteurs, les savoirs et savoir-faire se cristallisent certes autour de la conception, du suivi et de la réalisation opérationnelle d'un projet culturel, mais comprennent aussi l'expertise administrative et financière (gestion des contrats, des budgets...) et la capacité à créer et à animer un réseau de partenaires. Là encore, la gamme des compétences déborde largement le périmètre traditionnel de la médiation.

De même, les concepteurs et face au public à dominante contenus doivent, dans l'exercice de leur activité, maîtriser les savoirs et savoir-faire liés à la pratique artistique ou scientifique, alors que ceux à dominante publics mobilisent des connaissances relatives aux différents publics ou à l'élaboration de partenariats avec des structures liées à ces publics (écoles pour les scolaires, établissements pénitentiaires, etc.).

La spécificité des compétences des différentes populations de médiateurs pose la question de la polyvalence : la configuration «polyvalence», dans laquelle les différents pôles de médiation sont réunis au sein d'une même catégorie de personnel, est-elle viable ? Force est de constater que plusieurs institutions culturelles qui ont fait le choix de cette configuration (voir graphique 3 ) ont rencontré de telles difficultés qu'elles ont presque toujours abouti, dans un terme plus ou moins long, à la transformation de la configuration retenue, souvent en distinguant les deux pôles, conception et animation face au public. Il s'agit sans doute de deux espaces d'expertise et de savoir-faire largement disjoints (d'où les propos souvent entendus : les concepteurs ne passent pas toujours bien auprès du public, les spécialistes sont rarement des bons médiateurs), de plus l'articulation entre ces différents espaces nécessite d'être accompagnée de dispositifs de capitalisation et d'apprentissage, alors qu'elle est souvent laissée à l'initiative personnelle des médiateurs.

\section{Dynamique des compétences et organisation}

Il existe nécessairement un écart entre la configuration type et la configuration réelle. Dans cet écart se loge une dynamique des compétences, nécessaire pour faire face aux situations de médiation réellement rencontrées, qui relève le plus souvent d'un registre individuel: généralement chaque médiateur se forme par l'expérience pour acquérir les compétences manquantes. Il existe donc une dynamique spontanée qu'on peut qualifier d'ordinaire par rapport à des mouvements de réorganisation qui s'apparentent davantage à des changements de configuration plus stratégiques. L'exemple ci-contre illustre les implications d'un changement stratégique.

\section{Des voies de professionnalisation différentes}

Quelles sont les formes d'insertion professionnelle observées chez les différentes catégories de médiateurs?

\section{Trajectoires professionnelles et formes de mobilité}

L'insertion professionnelle des médiateurs apparaît problématique : beaucoup d'entre eux, quelle que soit la configuration professionnelle dans laquelle ils s'inscrivent, ren-

\section{Dynamique des compétences et professionnalisation au musée de $\mathbf{C}$.}

Le projet de construction d'un nouveau bâtiment et l'arrivée d'un nouveau directeur modifient l'organisation de l'activité de médiation au sein de ce musée et conduisent à mettre davantage l'accent sur la politique des publics.

Alors que, jusqu'alors, les médiateurs jouissaient d'une grande liberté d'action mais étaient peu impliqués dans la vie de l'institution, restant isolés par rapport au reste des activités (notamment la conception des expositions), le changement d'orientation leur fait perdre cette autonomie et modifie assez profondément leur champ d'action. Le fonds permanent des collections disparaît et de nouvelles expositions temporaires thématiques, pluridisciplinaires, et surtout plus transversales, prennent la place des expositions scientifiques traditionnelles. Une période difficile s'engage pour les médiateurs, qui manquent de connaissances sur ces nouveaux sujets, pour lesquels on leur demande non plus de transmettre des connaissances portées par les collections mais de jouer un rôle d'interface avec les publics, dans lequel l'objet devient prétexte pour tenir un discours qui, lui, devient premier.

À la fin de leur contrat emploi-jeunes, seuls trois médiateurs sur les huit présents à l'origine restent en place. Le musée fait alors appel à de nouveaux profils, plus généralistes (histoire, histoire des sciences...), mieux formés aux politiques des publics. Pour ceux qui restent, la question du statut se pose. Le musée les encourage à se présenter aux concours de la fonction publique territoriale, malheureusement peu adaptés à ces profils. Deux d'entre eux bénéficient de la transformation de leur contrat en $\mathrm{CDI}$, qui les maintient néanmoins, selon eux, dans une certaine forme de précarité. Seule la réussite au concours leur conférerait non seulement un statut, mais une véritable position dans l'institution.

Dernièrement, la direction du musée a redéfini le projet d'une organisation de la médiation entièrement remaniée, reposant sur trois populations hiérarchisées:

- 3 cadres $A$ « chargés de projet de médiation culturelle » définiraient la politique des publics, sur trois champs: le public scolaire, le public éloigné (dont les handicapés) et le public familial. Cette fonction ne serait pas ouverte aux médiateurs en fonction aujourd'hui ;

- 6 médiateurs de catégorie B («assistants qualifiés ») seraient chargés, sous la responsabilité des précédents, de concevoir les animations (trames de visite, tests sur le terrain, évaluation...); - 15 médiateurs étudiants (qualifiés d' « animateurs »), formés et encadrés par la catégorie précédente, assureraient les animations prédéfinies face au public. II s'agirait d'étudiants en dernière année d'études (master de médiation culturelle, de sciences dures ou sciences humaines...), effectuant un stage d'animation d'un an qui leur apporterait une formation professionnalisante.

On passe ainsi d'une configuration "polyvalence " à une configuration "bureau des méthodes " (voire même une configuration "la tête et les jambes", selon la capacité des étudiants à construire le contenu de leur intervention). 
contrent des difficultés pour intégrer l'espace professionnel puis pour y évoluer. Si l'absence de reconnaissance et d'explicitation du champ de compétences des médiateurs explique en partie ce constat, d'autres éléments contribuent à le renforcer. Nombre d'actions relevant de la gestion des ressources humaines (recrutement, mobilité, promotion...) dépendent des représentations relatives aux populations destinataires, et notamment de l'identification préalable de leur rôle. Or la population des médiateurs ne bénéficie pas d'une image structurée (situation souvent précaire, absence de statut...), non seulement au niveau national, mais au sein même de chaque institution culturelle.

Si l'on observe la professionnalisation d'un point de vue organisationnel - au sens des processus et leviers internes par lesquels les médiateurs acquièrent un corpus d'intervention les rendant capables de se déployer dans l'organisation (corpus de savoirs, repérage et positionnement dans l'organisation, coordination avec les autres professionnels du lieu...) -, il apparaît que les signes extérieurs de professionnalisation ne pèsent pas plus que les signes intérieurs, c'est-à-dire organisationnels. Ainsi, les guides conférenciers sont formellement une profession mais ne bénéficient que d'une reconnaissance limitée, du fait de leur positionnement aux côtés de concepteurs (par exemple dans le cas de certaines configurations plates « entre connaisseurs » rencontrées dans le secteur du patrimoine). Plus généralement, on observe que les médiateurs en face-à-face avec le public, tout particulièrement, subissent un confinement organisationnel sclérosant, dont les manifestations sont multiples (peu conviés aux réunions, interventions pensées en bout de course de la chaîne organisationnelle, absence de bureaux, etc.).

La mobilité est limitée par l'absence de reconnaissance des médiateurs : dans l'échantillon des personnes rencontrées au cours de l'enquête, la mobilité des animateurs en face-à-face avec le public, orientés vers les contenus aussi bien que vers les publics, est très faible. On peut relier ce constat à une caractéristique majeure du marché du travail dans ce secteur, très tendu. Pour une offre d'emploi, des dizaines de médiateurs, expérimentés ou non, font acte de candidature. Dans un tel contexte, les médiateurs en poste, quelle que soit leur spécialisation (conception ou public), hésitent à quitter leur emploi. Cela vaut également pour les vacataires, dont le statut d'emploi est plus précaire encore, mais qui apprécient de bénéficier d'un emploi, même faiblement rémunérateur, situé approximativement dans leur champ de compétence.

Dans le cas des animateurs orientés vers les contenus, cette situation s'explique d'autant plus qu'il s'agit souvent d'artistes qui se satisfont d'une activité de médiation, complémentaire à leur activité artistique. Absence de mobilité, professionnalisation lacunaire, confinement organisationnel se traduisent par la difficile acquisition de nouvelles expertises et aboutissent à une certaine démotivation. On aurait pu penser que des animateurs ou concepteurs très spécialisés étaient plus mobiles, mais il n'en est rien : leur compétence pointue peut aussi constituer un frein à la mobilité : ni mobilité hiérarchique (ils n'ont pas forcément les compétences ni le désir d'encadrer des équipes et d'abandonner la fréquentation assidue de leurs thèmes d'expertise), ni mobilité interorganisationnelle (car il leur est impossible de retrouver une institution correspondant à leur profil souvent très spécifique). De même, certains concepteurs orientés vers les publics développent un réseau avec lequel ils travaillent localement, qui leur confère un actif stratégique important pour leur institution, mais celuici s'avère souvent difficilement exportable. La mobilité semble en revanche plus élevée pour les concepteurs, leur bagage statutaire et leur corpus de connaissances étant manifestement mieux reconnus.

\section{Mobilité et configurations professionnelles}

L'étude de la mobilité peut s'envisager au plan organisationnel mais aussi entre configurations : elle permet d'observer si le passage au sein d'une configuration permet d'acquérir un corpus de connaissances et d'intervention consistant et reconnu au-delà de l'institution d'origine, favorisant la mobilité vers d'autres types d'emplois ou d'autres configurations. Or, l'étude montre que les cas de mobilité concernent essentiellement des changements d'emplois, positionnés sur un même type de configuration. Cette mobilité est sans doute facilitée pour les concepteurs à dominante publics du fait de la généralisation de la configuration «la tête et les jambes » qui favorise du coup la reconnaissance de leurs compétences et augmente leur aire d'employabilité par rapport à des profils développés dans des configurations moins répandues.

On observe quelques rares cas de passage de l'animation vers la conception : une guide conférencière et une animatrice en face-à-face avec le public dans un centre d'art contemporain devenues toutes deux conceptrices de politique de médiation dans une collectivité ; un animateur en face-à-face avec le public d'un centre d'art contemporain devenu concepteur orienté public dans un autre centre d'art; enfin, une conceptrice animatrice polyvalente d'un centre d'art contemporain devenue conceptrice.

Si le passage du face-à-face avec le public à la conception reste délicat, certaines configurations, qui valorisent la polyvalence, favorisent cette évolution.

\section{Quelles leçons tirer de cette analyse en termes de gestion des ressources humaines?}

Les médiateurs, après avoir été comme «convoqués » par les politiques culturelles, apparaissent ainsi abandonnés par le silence de l'appareil gestionnaire des institutions culturelles, qui omettent d'élaborer un véritable pilotage de cette activité : recrutement sans réflexion sur leur insertion dans l'organisation, absence d'identification des compétences requises, évolution de carrières sans construction de filières professionnelles ni mise en place de formations spécifiques ou de modalités de gestion des compétences, évolution de l'activité vers de nouvelles frontières sans leur affecter de véritables assises professionnelles. Créer un nouveau réceptacle d'emplois, tels que les emplois-jeunes, impulser la création ou le renforcement de nouvelles activités ne suffisent pas à créer de véritables espaces professionnels pour les acteurs de ces activités, et doivent être complétés à l'avenir par un accompagnement gestionnaire.

Divers outils de gestion des ressources humaines peuvent être mobilisés dans cette perspective. Les outils statiques sont les plus usités : depuis la mise en place d'une 
véritable politique de l'évaluation et donc de capitalisation des connaissances nées pendant l'acte de médiation, à la constitution de fiches de description des emplois concernés. À cet égard, il existe d'ores et déjà des tentatives d'élaboration de répertoires de compétences de la médiation. On peut notamment citer l'exemple du répertoire du Centre national de l'éducation à distance (Cned), conçu pour établir des bilans individuels de compétences, qui fut à l'origine de la réalisation de répertoires plus spécifiques à certains champs artistiques comme l'art contemporain. Un tel outil offre un premier niveau de représentation des activités et des compétences déployées dans une configuration donnée. Il permet notamment d'identifier des types de métiers qu'il est possible de retrouver dans différentes configurations. Mais, par construction, il ne s'attache qu'à décrire des objets relevant des ressources humaines (emplois, métiers, professions) qui sont des entités isolées, sans en appréhender la dimension collective, organisationnelle; par ailleurs, d'un point de vue des ressources humaines, il est important de prendre aussi en compte les mobilités et évolutions de carrière. Une cartographie dynamique des configurations professionnelles pourrait être élaborée, intégrant des scénarios d'évolution des activités de médiation culturelle, en prenant appui sur les résultats de cette étude.

\section{Conclusion : \\ LA MÉDIATION CULTURELLE, UN PAYSAGE EN TROMPE-L'GEIL ?}

La médiation est aujourd'hui une évidence partagée pour l'ensemble des structures culturelles. Après trente ans de développement, l'intérêt d'offrir aux publics les moyens d'accéder aux œuvres, de développer des capacités critiques et artistiques est considéré comme essentiel. Car l'ambition de la médiation est bien là, portée par des voix diverses, théoriques et doctrinales : contribuer à régénérer l'action culturelle, en allant même jusqu'à repenser le statut des institutions culturelles les plus patrimoniales pour en faire, en tant qu'institutions mêmes, des médiateurs.

Ce n'est pas seulement par des descriptions abstraites des ambitions et des compétences que la médiation atteindra l'objectif de démocratisation culturelle, mais bien en s'incarnant, dans des organisations et dans des formes de ressources humaines adaptées. C'est dans ce double processus que se mesure le degré d'achèvement d'un projet de médiation. Or, de ce point de vue, le paysage de la médiation apparaît plus fragmenté que l'on pouvait s'y attendre. D'un continent théorique, on passe ainsi, en se transportant du lieu des discours à celui des pratiques, à une fragile économie d'archipel.

Au plan des pratiques, la médiation s'inscrit tout d'abord dans des configurations professionnelles variées, bien que certaines semblent privilégiées par les structures culturelles, quel que soit leur secteur. La variété de ces configurations traduit la contingence organisationnelle des activités de médiation. La médiation culturelle ne se développe pas de la même manière selon la structure dans laquelle elle s'insère, les types de publics auxquels elle s'adresse, la nature des relations qu'elle entend tisser avec eux. Ce fait organisationnel, d'apparence banal, est un élément central pour comprendre l'état de relative dispersion des activités de médiation, et parfois leur position marginale.

Il est en outre intimement lié à un deuxième processus d'incarnation des activités. Derrière la figure du médiateur se cache en réalité une large gamme de profils. Quelles que soient les organisations étudiées, un clivage très prononcé se manifeste entre, d'un côté, ceux que l'on a appelés des concepteurs producteurs et, de l'autre, des animateurs en situation de face-à-face avec les publics. Le positionnement de ces populations (notamment en raison des compétences à dominante contenus ou publics) et les relations qu'elles entretiennent entre elles et avec les autres acteurs de la structures, peuvent connaître des variations sensibles d'une configuration à l'autre. Néanmoins, il apparaît globalement que les formes d'exercice professionnel des animateurs en situation de face-à-face avec le public sont moins abouties (en termes de statut, de mobilité, de reconnaissance, etc.) que celles des concepteurs producteurs. Enfin, la professionnalisation de ces deux populations est encore fragile ou inachevée.

Le champ de la médiation a connu des extensions vers de nouveaux territoires, précisément ceux de l'action territoriale, et les collectivités locales ne sont pas étrangères à cette évolution. L'action territoriale recouvre un ensemble assez vaste d'actions, fortement soutenues par différentes politiques publiques, mais n'a pas contribué à générer le mouvement de professionnalisation des métiers de la médiation auquel on aurait pu s'attendre. Plus généralement, la médiation culturelle est aujourd'hui l'exemple type d'une contradiction de la gestion publique qui se manifeste à deux niveaux.

Alors que les discours ne cessent de réaffirmer que les publics sont au centre de l'action, les activités de service face à ces mêmes publics restent toujours extrêmement mal décrites et par conséquent faiblement reconnues. Les activités de conception de dispositifs subissent, à un niveau moindre, le même traitement.

Alors que l'on prône sans cesse une professionnalisation accrue des activités de médiation, elles ne parviennent pas à être reconnues. Qu'il s'agisse de la prise en compte des publics ou de sa professionnalisation, l'étude des évolutions des emplois de la médiation culturelle révèle un grand écart persistant entre discours et pratiques. À quelles conditions peut-on alors espérer un changement plus radical dans la situation de la médiation, une mise en cohérence des discours et des pratiques?

Les pratiques de médiation pourront s'approcher des ambitions théoriques affichées, lorsqu'elles deviendront un véritable objet de gestion.

Le diagnostic réalisé plaide fortement en faveur de la mise en œuvre d'une politique d'accompagnement et de structuration de ces activités en termes de ressources humaines, afin que soient clairement identifiées (en préalable à la définition de véritables filières professionnelles) les compétences requises, que soient précisées les modalités de gestion de ces compétences, et que soit mieux définie la place de ces activités au sein des organisations. 


\section{Éléments de méthodologie}

Réalisée par le centre de gestion scientifique de l'École des mines de Paris, l'étude s'est déroulée tout au long de l'année 2008. De nature qualitative, reposant sur la réalisation d'une centaine d'entretiens en face-à-face et sur des observations in situ, elle s'est déployée sur plusieurs territoires contrastés choisis dans trois régions françaises (Île-de-France, Nord - Pas-deCalais, Rhône-Alpes). L'enquête de terrain a consisté en: - une consultation de plusieurs experts de la médiation;

- la mobilisation, à plusieurs temps forts du déroulement de l'enquête, d'un groupe focus composé de représentants des organisations professionnelles du domaine de la médiation et d'experts des différents domaines culturels, chargés de réagir aux résultats intermédiaires, d'exprimer les grands débats et enjeux auxquels est soumise la profession, et de dégager les grandes tendances d'évolution;

- la réalisation, au sein de 48 structures employeuses différentes, de 103 entretiens en face-à-face auprès de différentes catégories d'acteurs:

- responsables sectoriels (responsables dans des directions sectorielles du ministère de la Culture ou en DRAC, dans des collectivités territoriales, associations professionnelles, etc.) ;
- responsables de politiques ou de lieux culturels (directeurs des affaires culturelles, directeurs d'institution culturelle, etc.);

- responsables de services de médiation;

- chargés de conception des actions de médiation;

- agents réalisant eux-mêmes des actions de médiation en face-à-face avec le public (visites, conférences, ateliers, etc.).

Ont ainsi été enquêtés : 48 lieux (103 entretiens)...

-... relevant des domaines culturels suivants: musées (9 structures), patrimoine (3), art contemporain (12), bibliothèques et livre (4), spectacle vivant (12), cinéma (3), autres (5)...

-... dans des structures employeuses de taille variée : moins de 10 permanents (10 structures), de 10 à 100 permanents (15), plus de 100 permanents (9)...

-... dans trois régions (Île-de-France, Nord - Pas-de-Calais, Rhône-Alpes). Des entretiens ont toutefois été réalisés également en dehors de ces trois territoires régionaux, au sein d'institutions et de structures présentant une approche originale de la médiation.

\section{RÉSUMÉ}

Au cours des 30 dernières années, les activités de médiation culturelle ont connu un essor continu dans l'ensemble des domaines artistiques et culturels, au sein des institutions et sur les territoires, notamment sous l'impulsion des politiques publiques de la culture, de la ville et de l'emploi. Pour autant, les activités de médiation ne constituent pas aujourd'hui un ensemble homogène ; le métier et les compétences, divers selon les établissements et les secteurs, restent peu reconnus. Au moyen d'une méthode inédite qui se décline sous la forme de 6 configurations professionnelles, l'étude dresse un état des lieux actualisé des situations professionnelles rencontrées (contenus d'activité, statuts d'emploi, conditions d'accès et identités professionnelles) et identifie l'enjeu majeur que constitue la gestion des ressources humaines pour la professionnalisation des activités de médiation.

\section{ABSTRACT}

Over the last 30 years, work within cultural events promotion has been growing steadily in all cultural and artistic fields, within institutions and across regions, driven by public policies on cultural, civic and business life. As such, cultural promotion now no longer covers a consistent range of activities; the profession and its related skills vary widely across institutions and sectors and with little recognition. Using a completely new method which designates 6 separate professional categories, this study gives an up-to-date review of professional situations covered (activity, employment status, entry conditions and professional identity) and identifies the major issue of human resource management in the professionalisation of cultural promotion activities.

Tous les documents publiés par le DEPs sont téléchargeables sur http://www.culture.gouv.fr/deps

Le DEPS n'assurant pas de diffusion physique de ces documents, nous vous proposons de vous informer régulièrement des parutions par message électronique. Pour ce faire, merci de bien vouloir nous communiquer votre courriel à l'adresse contact.deps@culture.gouv.fr 